\title{
Ab-initio study of electronic and magneto-optical properties of InAs:Mn
}

\author{
Elena Gan'shina ${ }^{1, *}$, Erkin Kulatov $^{2,4}$, Leonard Golik ${ }^{3}$, Zoya Kun'kova ${ }^{3}$, Yurii Uspenskii ${ }^{4}$, Georgy Zykov ${ }^{1}$, Ye Yuan ${ }^{5}$, \\ andShengqiangZhou \\ ${ }^{1}$ Department of Physics, Moscow State University, Moscow 119991, Russia \\ ${ }^{2}$ Prokhorov General Physics Institute, RAS, Moscow 119991, Russia \\ ${ }^{3}$ Institute of Radioengineering and Electronics, RAS, Fryazino 141190, Russia \\ ${ }^{4}$ Lebedev Physical Institute, RAS, Moscow 119991, Russia \\ ${ }^{5}$ Helmholtz-Zentrum Dresden-Rossendorf, Institute of Ion Beam Physics and Materials Research, 01328 Dresden, Germany
}

\begin{abstract}
Energy difference between the ferromagnetic and antiferromagnetic collinear orderings has been calculated for the uniform and dimer Mn-pair geometries in order to find the ground state distribution of the $\mathrm{Mn}$ atoms in InAs host. We find the preference of the dimer ferromagnetic configuration of Mn dopants and an importance of optimizing the atomic site positions. The frequency-dependent optical and magnetooptical properties, namely the dielectric tensor (on- and off-diagonal components), the electron energy loss spectra, and the transversal Kerr effect (TKE), are calculated. Calculated TKE resonance in $\operatorname{In}_{1-\mathrm{x}} \mathrm{Mn}_{\mathrm{x}} \mathrm{As}$ $(\mathrm{x}=0.0625)$ is found to be in good agreement with corresponding experimental magneto-optical spectra. The origin of the large TKE is discussed.
\end{abstract}

\section{Introduction}

Diluted magnetic semiconductors (DMS) are considered as promising materials for spintronics. However the Curie temperature $\left(\mathrm{T}_{\mathrm{C}}\right)$ of the magnetic semiconductors is below room temperature and this fact limits their practical application. To increase $T_{C}$ one should increase concentration of magnetic impurity in host semiconductor. In DMS the concentration is limited by low solubility of magnetic impurities in these materials. Exchange interaction between neighbouring $\mathrm{Mn}$ ions would be stronger in semiconductor based structures with inhomogeneous distribution of $\mathrm{Mn}$ ions, and achieved Curie temperature in such structures would be significantly higher than in DMS with uniform distribution of magnetic dopants. In this paper we study electronic and magneto-optical properties of InAs:Mn DMSregarding its possible application in spintronics.

\section{Calculational method}

Our calculations are performed using the relativistic Augmented Plane Wave + local orbital (APW + lo) method as implemented in WIEN2k code [1]. The exchange-correlation effects are described within the Generalized Gradient Approximation (GGA)of density functional theory [2]. Density-functional calculations presented here are performed within the supercell $(2 \times 2 \times 2$ cubic zinc blende unit cell), corresponding to 64 atoms.
One or two Mn atoms are included in the supercell. We consider substitutional doping where In host atoms are replaced by $\mathrm{Mn}$ ones. Thus, the Mn concentration in our calculations varies in the experimentally relevant range $\left(\mathrm{x}_{\mathrm{Mn}}=0.0312\right.$ and 0.0625$)$. Experimental lattice parameter of InAs host $\left(a_{0}=6.058 \AA\right)$ [3] is also used for InAs:Mn DMS's. The $4 \mathrm{~d}$ states of In and $3 \mathrm{~d}$ states of As are included in the APW basis set and treated as the valence orbitals. Converged results are obtained at the cut-off energy for the interstitial plane-wave expansion of $125 \mathrm{eV}$. Relaxations of all atomic positions have been included in our calculations. The total energy difference between ferromagnetic (FM) and antiferromagnetic (AFM) collinear orderings has been calculated for the uniform and dimer Mn-pair geometries in order to find the ground state distribution of the $\mathrm{Mn}$ atoms in InAs host. The magneto-optical transversal Kerr effect of $\operatorname{In}_{1-}$ ${ }_{x} \mathrm{Mn}_{\mathrm{x}} \mathrm{As}(\mathrm{x}=0.0625)$ is calculated in the framework of the relativistic WIEN2k method [1], which takes into account the effect of spin-orbit coupling in the second variational procedure.

\section{Results and Discussion}

Optimization of the positions of all atoms in a supercell reveals that in InAs:Mn the bond length Mn-As shrinks by $2.5 \%$ in comparison with the unrelaxed case. Relaxation effects for the bonds Mn-In were found to be negligible (contraction by $0.1 \%$ ). The calculated relativistic density of states (DOS) for an energetically 
important Mn dimmer configuration is shown in Fig. 1 for InAs:MnMn in the FM dimer and separate configurations and with $\mathrm{Mn}$ concentration equal to 0.0625 . It is seen that InAs: Mn in both configurations is close to the half-metallic state. The main feature of shown DOS's is the large exchange splitting of the $3 \mathrm{~d}$ states of $\mathrm{Mn}(\sim 4 \mathrm{eV})$.We find the preference of the dimer configuration of Mn dopants, i.e. the configuration
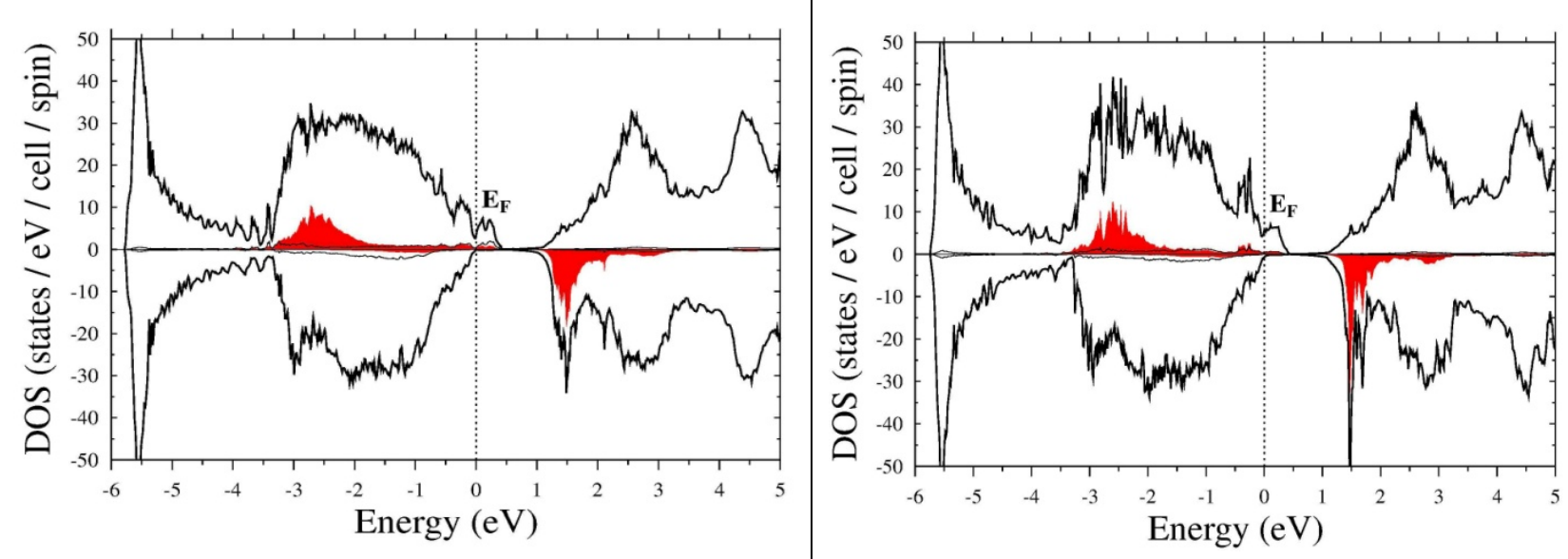

Fig. 1.Relativistic DOS in $\operatorname{In}_{1-\mathrm{x}} \mathrm{Mn}_{\mathrm{x}} \mathrm{As}$ for the dimer configuration (left panel) and for the separate configuration (right panel). Bold line - total DOS, shaded red region $-3 \mathrm{~d}$ Mn partial DOS, thin line $-4 \mathrm{p}$ As partial DOS, $\mathrm{E}_{\mathrm{F}}$ means the Fermi level.

Table 1.The AFM and FM energy differences $\left(\Delta\left(E_{\mathbf{A F M}}-E_{F M}\right)\right)$, total and local (in parentheses) magnetic moments $\left(M_{F M}, M_{A F M}\right)$ in the FM and AFM phases, $\Delta(\mathrm{FM})$ and $\Delta(\mathrm{AFM})$ are the energies of the FM and AFM phases with respect to the energy of the FM dimer.

\begin{tabular}{|c|c|c|c|c|}
\hline $\begin{array}{c}\text { Configuration of } \\
\text { Mn atoms }\end{array}$ & $\begin{array}{c}\Delta\left(\mathrm{E}_{\mathrm{AFM}}-\mathrm{E}_{\mathrm{FM}}\right), \\
\mathrm{meV} / \mathrm{Mn}\end{array}$ & $\begin{array}{c}\mathrm{M}_{\mathrm{FM}}, \\
\mu_{\mathrm{B}} / \mathrm{cell}(/ \mathrm{Mn})\end{array}$ & $\begin{array}{c}\mathrm{M}_{\mathrm{AFM}}, \\
\mu_{\mathrm{B}} / \mathrm{cell}(/ \mathrm{Mn})\end{array}$ & $\begin{array}{c}\Delta(\mathrm{FM}) \\
\mathrm{meV} / \mathrm{Mn}\end{array}$ \\
\hline close (dimer) & +233 & $8.08(+4.08)$ & $0.002( \pm 4.11)$ & 0 \\
\hline intermediate & +218 & $8.08(+4.09)$ & $0.003( \pm 4.13)$ & $+120+338$ \\
\hline separate & +55 & $8.13(+4.11)$ & $0 \quad( \pm 4.14)$ & $+125+180$ \\
\hline
\end{tabular}

where the Mn atoms share the same neighbouring As site is always found to be energetically most favorable (see the 5-th column of Table 1). So our calculations show that clustering effect is energetically favorable in agreement with the experimental results [4]. Also we found that the FM order is the most stable magnetic state (Table 1, the2-nd column).An interesting feature of DOS is a narrow unoccupied part of the valence band, which is placed just above the Fermi level. This area of $0.4 \mathrm{eV}$ width is separated from the conducting band by the quasigap of $0.9 \mathrm{eV}$ width. As is shown below, electron transitions from occupied states to this unoccupied part of the valence band and to the conduction band (across the quasigap) form important spectral features in the dielectric tensor and energy loss spectra.

It is known [5] that TKE is determined by both magnetooptical and optical parameters of a medium. The TKE value, $\delta_{\mathrm{p}}=\left(\mathrm{I}(\mathrm{H})-\mathrm{I}_{0}\right) / \mathrm{I}_{0}$, is the relative change in intensity of the reflected light caused by the magnetization of a sample in an external magnetic field whose direction is transverse to the plane of the light incidence. TKE observed at the angle of incidence $\varphi$ can be expressed by the off- and on-diagonal components of the dielectric function as

$$
\begin{gathered}
\delta_{\mathrm{p}}=2 \sin (2 \varphi)\left(\mathrm{A} /\left(\mathrm{A}^{2}+\mathrm{B}^{2}\right)\right) \cdot \varepsilon_{1}^{\prime}+\left(\mathrm{B} /\left(\mathrm{A}^{2}+\mathrm{B}^{2}\right)\right) \cdot \varepsilon_{2}^{\prime}= \\
\mathrm{a} \cdot \varepsilon^{\prime}{ }_{1}+\mathrm{b} \cdot \varepsilon_{2}^{\prime}{ }_{2},
\end{gathered}
$$

where $\varepsilon_{1}^{\prime}$ and $\varepsilon_{2}^{\prime}$ are the dispersive and absorptive parts of the off-diagonal dielectric tensor, respectively, while $\mathrm{A}=\varepsilon_{2} \cdot\left(2 \varepsilon_{1} \cdot \cos ^{2} \varphi-1\right)$ and $\mathrm{B}=\left(\varepsilon_{2}^{2}-\varepsilon_{1}^{2}\right) \cdot \cos ^{2} \varphi+\varepsilon_{1}-\sin ^{2} \varphi$ are the functions of the on-diagonal components of the dielectric tensor $\varepsilon_{1}$ and $\varepsilon_{2}$. Fig. 2 displays calculated TKE spectra, which show marked features in the infrared region. The energy position of our calculated $\mathrm{MO}$ resonance in $\operatorname{In}_{1-\mathrm{x}} \mathrm{Mn}_{\mathrm{x}} \mathrm{As}(\mathrm{x}=0.0625)$ are found to be in good agreement with the corresponding experimental TKE spectra (obtained by us in[6]) at $\sim 0.7 \mathrm{eV}$.

Spectra of calculated and off- and on-diagonal components of the dielectric tensor and electron energy loss spectrum are shown in Figs. 3, 4.Although the MO property is a rather complicated function of the ondiagonal and off-diagonal components of the optical conductivity tensor, our calculation provides a clear insight about its origin(see Figs. 2-4). The special feature 
of DOS noted above forms intensive electron transitions with $\hbar \omega<0.4 \mathrm{eV}$ (to the unoccupied part of the valence band) and with $\hbar \omega>1.3 \mathrm{eV}$ (to the conduction gap) and a peak of $\varepsilon_{2}$ at $2.2 \mathrm{eV}$ (the left panel of Fig. 4).Large TKE observed in InAs:Mn can be understood as a combined effect of the on- and off-diagonal components, nearly maximal exchange splitting of the Mn3dstates and large

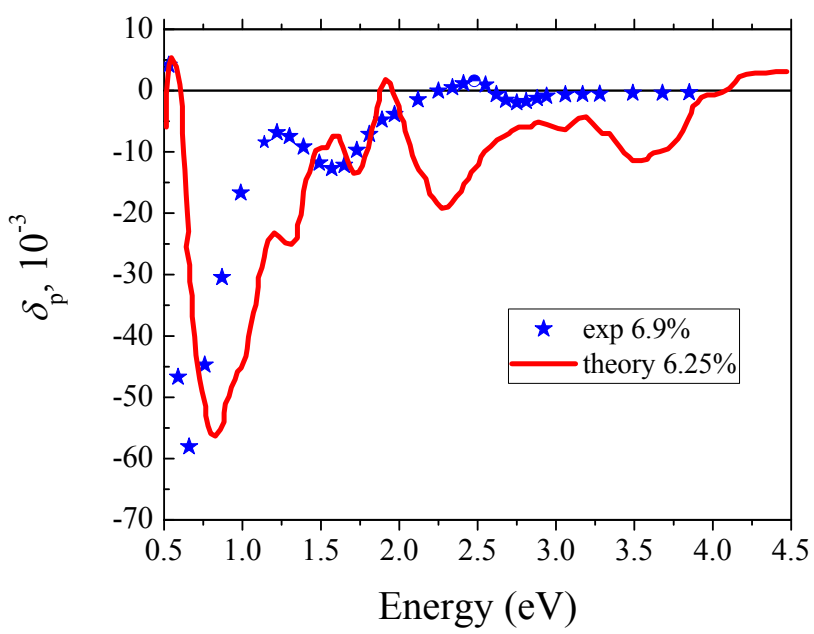

spin-orbit coupling. Additional enhancement of the $0.7 \mathrm{eV}$ TKE peak comes from the plasma resonance at about $0.65 \mathrm{eV}$, which occurs in our calculated electron energy loss spectrum (EELS) (the right panel of Fig. 4) near the minimum of $\varepsilon_{2}$.

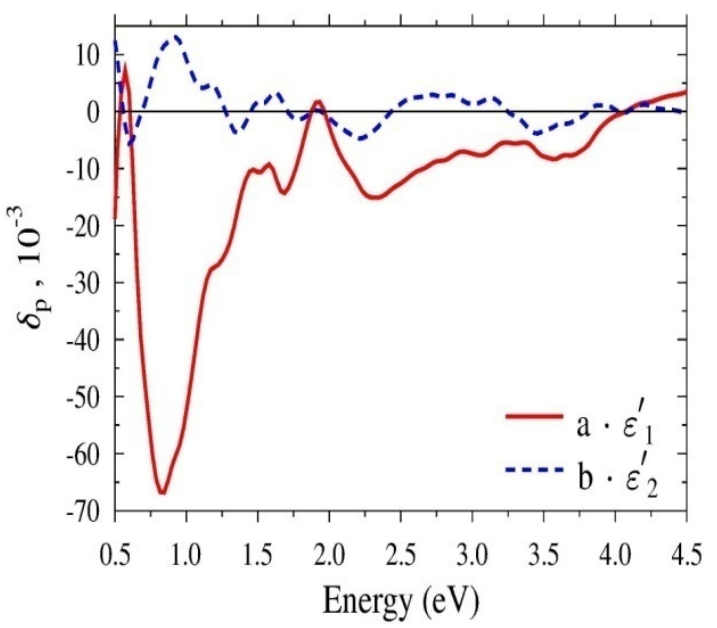

Fig. 2.Left panel:calculated TKE in InAs:Mnfor the dimer configuration. Symbols are the experimental TKE data for InAs: Mn (x= 0.069 ) from[6]. Angle of light incidence $\varphi=66^{\circ}$. Right panel shows decomposition of $\delta_{\mathrm{p}}$ into the sum of products (see Eq. (1)).
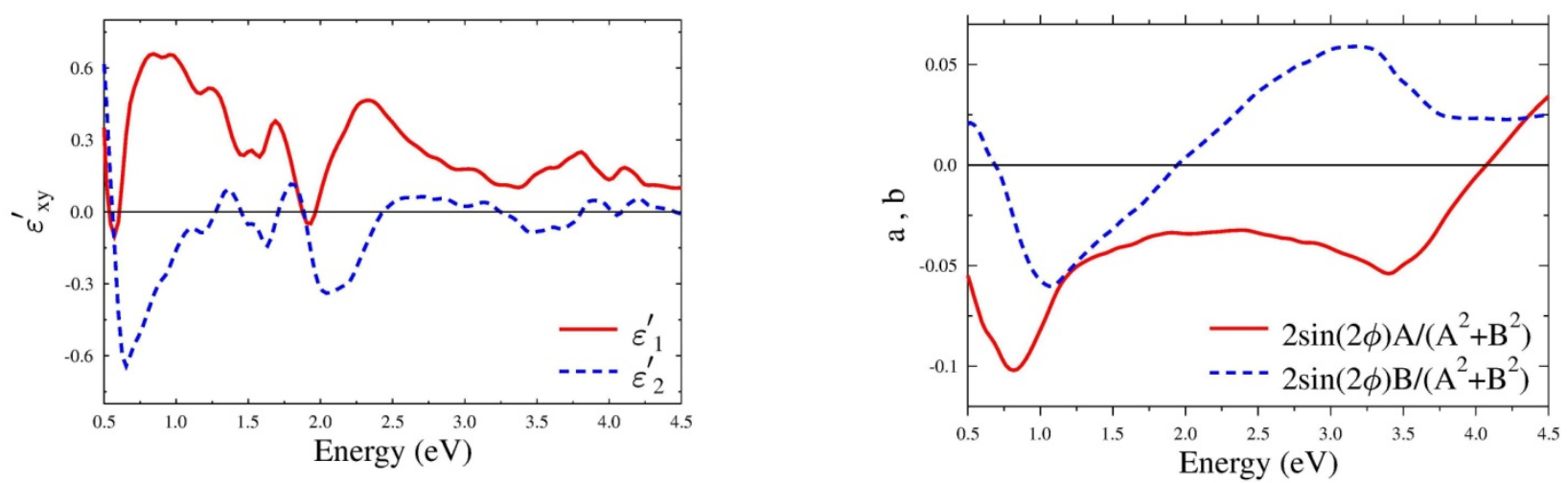

Fig. 3. Calculated off-diagonal components of the dielectric tensor $\varepsilon_{\mathrm{xy}}^{\prime}=\varepsilon^{\prime}{ }_{1}+\mathrm{i} \varepsilon_{2}{ }_{2}($ left panel) and $\mathbf{a}$, b factors (see Eq. (1)) (right panel).
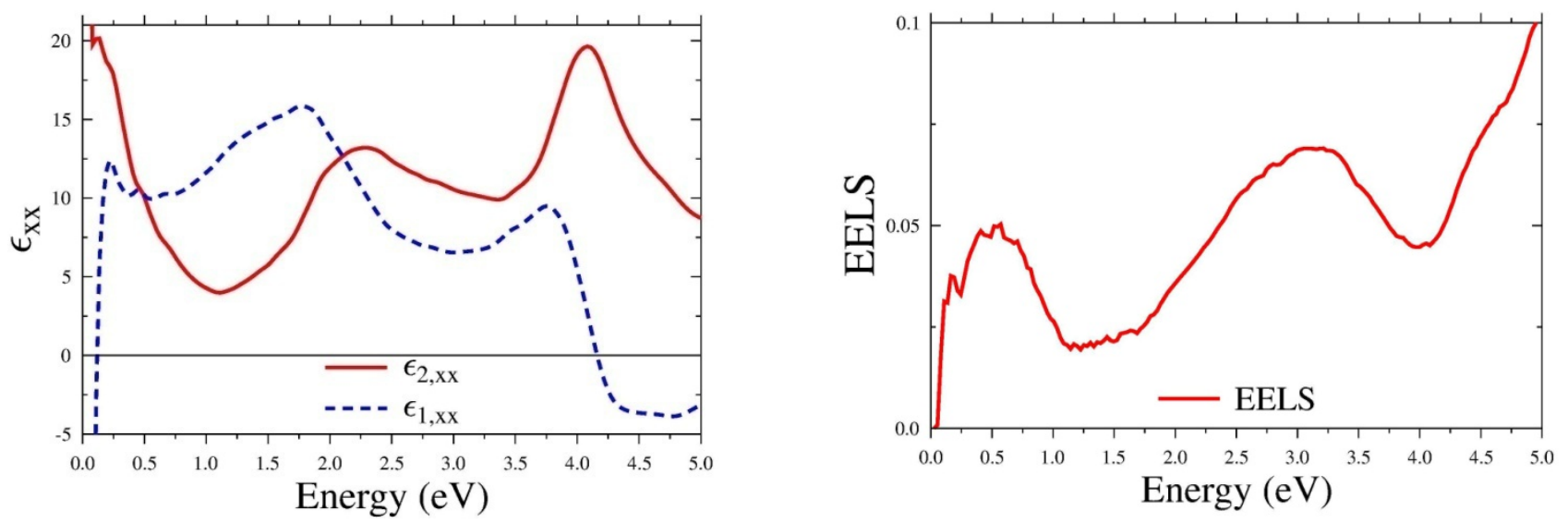

Fig. 4.Calculatedon-diagonal components of the dielectric tensor $\varepsilon_{\mathrm{xx}}=\varepsilon_{1}+\mathrm{i} \varepsilon_{2}$ (left panel) and electron energy loss spectrum (right panel). 


\section{Conclusion}

Based on the calculation of the relativistic density of states for InAs:Mn in the dimer and separate configurations is found that InAs: $\mathrm{Mn}$ in both configurations is close to the half-metallic state. But the dimer ferromagnetic configuration of $\mathrm{Mn}$ dopants is always found to be energetically most favorable. Also found that the FM order is the most stable magnetic state.

The frequency-dependent optical and magneto-optical properties, the electron energy loss spectra, on- and offdiagonal conductivity tensor components and the transversal Kerr effect (TKE) are calculated. The modeled TKE resonance in $\operatorname{In}_{1-\mathrm{x}} \mathrm{Mn}_{\mathrm{x}} \mathrm{As}(\mathrm{x}=0.0625)$ in near IK range is found to be in good agreement with the experimental magneto-optical spectrum. The additional enhancement of the $0.7 \mathrm{eV}$ TKE peak comes from the plasma resonance at about $0.65 \mathrm{eV}$, which occurs in the calculated electron energy loss spectrum.

Support by Russian Foundation for Basic Research No 15-0202077, 16-02-00612-a, 16-02-00024-a, 16-07-00798, and Program of Russian Academy of Sciences "Strongly correlated electrons in solids and structures" and "Basic investigations of nanotechnologies and nanomaterials" is acknowledged.

\section{References}

1. P. Blaha, K. Schwarz, G.K.H. Madsen, D. Kvasnicka, J. Luitz, Wien2k, An Augmented Plane Wave+Local Orbitals Program for Calculating Crystal Properties (Karlheinz Schwarz, Tech. Universitat Wien, Austria, 2001).

2. J.P. Perdew, S. Burke, M. Ernzerhof Phys. Rev. Lett. 77, 3865(1996).

3. NIMS Materials Database, National Institute for Materials Science, Tsukuba, Japan, http://crystaldb.nims.go.jp/crystdb.

4. N.D. Parashar, N. Rangaraju, V.K. Lazarov el al Phys. Rev. B 81, 115321(2010).

5. G.S. Krinchik and G.M Nurmukhamedov, Sov. Phys. JETP, 21, 22(1965).

6. Elena Gan'shina, Leonard Golik, Zoya Kun'kova, IgorBykov, Andrey Novikov, Alexander Rukovishnikov, Ye Yuan, GeorgyZykov, Roman Böttger, and Shengqiang Zhou, Japanese Journal of Applied Physics, 55,07MF02-1 (2016), http://doi.org/10.7567/JJAP.55.07MF02. 